\title{
Magnetoresistive Sensors for the Application in Biotechnology
}

\author{
Tsung-I Yin, Johannes Paul, Ronald Lehndorff \\ Sensitec GmbH, Hechtsheimer Str. 2,55131 Mainz, Germany \\ Tsung-I.Yin@sensitec.com \\ Johannes.Paul@sensitec.com \\ Ronald.Lehndorff@sensitec.com
}

\begin{abstract}
Magnetic field sensing is one of the main sensing mechanism for sensor technology. Magnetic sensor based on magnetoresistive (MR) effect have found import applications in biotechnoloy beyond information storage. This paper covers some of examples using MR sensors for biomagnetic signal detection, cardiac implantable electronic devices (CIED), and in-vitro-diagnostics (IVD) test with pointof-care (POC) application. There are plenty of research and market opportunities for MR sensors in biotechnology.
\end{abstract}

Keywords: magnetic field sensing, MR sensors, cardiac implantable electronic devices (CIED), biomagnetic signal detection, in-vitro-diagnostics (IVD), point-of-care (POC).

\section{Introduction}

Magnetic sensors are invented for more than 2000 years. The technology of magnetic sensors has been evolved driven by various need in market for improved sensor performance, smaller size, integration with electronic systems, and cheaper price. Depending on the demand of field sensing range, the magnetic sensors can be roughly divided into three categories-low field (less than 1 microgauss), medium field (1 microgauss to 10 gauss), and high field sensing (above 10 gauss) [1]. The low field sensors, such as Superconducting Quantum Interference Device (SQUID), search-coil, and fiber-optic magnetometers, are mainly used for medical applications and military surveillance. The medium field sensors, such as fluxgate and magnetoinductive magnetometers are suitable for detecting the earth's magnetic field. Most industrial sensors for high field sensing use permanent magnets (bias) as a source of the detected magnetic field.

Magnetic sensors have found important applications in biotechnology. One of typical applications is to sense the magnetic fields generated by physiological functions, e.g. neuronal signals and cardiac signals. Compared with the implanted electrodes to pick up voltage signal in living tissues, monitoring physiological signals by detecting the magnetic fields can be non-invasive and therefore prevent the issues in surgery and biocompatibility of implanted devices. Ultrasensitive magnetic sensor such as SQUID have been integrated in biomedical signal detection platforms for both magnetoencephalography (MEG) and magneto cardiography (MCG) [2].

Cardiac implantable electronic devices (CIED) such as pacemakers and implantable cardioverter defibrillators (ICD) have been widely used to treat the various forms of cardiac arrhythmias, i.e. abnormal heart rhythms. Based on the need of the patient, the therapy can be specifically tailored by programming the CIEDs. The use of magnets and magnet sensors in CIEDs is still widely used today to initiate the programming of the CIEDs [3].

Another recent research interest is to develop magnetic sensors-based platforms for in-vitrodiagnostics (IVD) test. A new generation of IVD test devices provide a point-of-care (POC) test alternative to centralized testing with large and automated high-throughput system, which requires for sample transportation and storage until testing, and many sets of reagents [4]. Devices for POC test must be simple to operate and low cost, while maintaining a multi-analytes, high-throughput, specific and sensitive assay. With the aid of small magnetic beads coated with biological molecules, DNA, or living cells for versatile biomedical assays, magnetic sensors which can detect small stray 
field generated by magnetic beads may open a new era for IVD and POC test $[2,5]$.

\section{Magnetoresistive (MR) Effect and MR Sensors Design}

Among different types of solid state magnetic sensors, magnetoresistive (MR) sensor has drawn particular attention to the low field sensing in biotechnology. The MR sensor is a collective term for the sensors based on MR effects, which show basically the change of a metal system's electric resistivity under the influence of an external magnetic field. Anisotropic MR (AMR) effect was first discovered by William Thomson (Lord Kevin) in 1857 by using different ferromagnetic samples [6]. The initial work showed only a small effect in low field, $2 \%$ resistance at room temperature. Tunnel MR (TMR) effect that occurred in a magnetic tunnel junction (MJT), which is a component consisting of two ferromagnet layers separated by a thin insulator, was discovered by Julliere in 1975 [7]. The relative change of resistance was around $14 \%$, and did not attract much attention at that time. Giant MR (GMR) effect was discovered in 1988 independently by the groups of Albert Fert of the University of ParisSud, France, and Peter Grünberg of Forschungszentrum Jülcih, Germany [8, 9]. Their remarkable findings showed that it was possible to generate resistance changes on a greater scale and therefore was recognized by the 2007 Nobel Prize in Physics later on. Understanding the potential of using GMR effect for magnetic sensors, Parkin and his colleagues in IBM Almaden Research Center investigated the possibility to realize GMR sensor using sputtering, a common hard drive manufacturing techniques at that time, instead of molecular beam epitaxy (MBE), a more expensive and complicated technique [10]. Their successful works lead to an ever since increasing storage density in hard drives. Since the year 2000, tunnel barriers of crystalline magnesium oxide $(\mathrm{MgO})$ have been developed for over $200 \%$ TMR at room temperature [11].

The construction of MR sensor using AMR, GMR, and TMR effect can be schematically depicted in Fig. 1. Since MR effect is sensitive to temperature variation in measurement environment, Wheatstone bridge is widely adopted for MR sensors design to reduce the temperature effect and noise. Depending on the number of "active" MR resistors, Wheatstone bridge can be categorized as single active bridge, half active bridge, and fully active bridge as shown in Fig. 2 (a). Fully active bridge provide full signal output as well as higher linearity of sensor compared with the half active bridge and single active bridge. To achieve fully active bridge in AMR sensors, barber pole structure has been adopted to vary the angle between current and external magnetic field in resistors 1-4 ((Fig. 2 (b)).Thick soft magnetic film has been integrated with GMR sensor to realize half active bridge (Fig. 2 (c)). In general, MR sensors offer a low-power-consumption, high accuracy, high spatial resolution, high sensitivity, and high reliability solution for magnetic field sensing. Fabrication process of MR sensors can also be easily integrated with standard IC fabrication process for advanced on-chip signal processing.

\section{MR Sensors for Biomagnetic Signal Detection}

The non-invasive biomagnetic signal detection platforms have been extensively investigated and utilized for studying the physiological functions of the brain and of the heart. The magnetic field amplitude generated by the neuronal signals and cardiac signals is approximately of the order of $50 \mathrm{fT}$ and $1 \mathrm{pT}$, respectively [2]. The most sensitive commercial systems for detecting those signals use extremely sensitive SQUIDs, which is capable to detect the signal down to 1 $\mathrm{fT} /(\mathrm{Hz})^{1 / 2}$. The SQUIDs, however, are limited by their operation temperature and the size of sensor area, which is proportional to its sensitivity [2].

Although the magnitude of biomagnetic signal is far beyond the detectable range of MR sensors in market, a mixed sensor design has been proposed by integrating a GMR sensor with a high-Tc superconductor YBCO as a fluxto-field transformer [12]. As shown in Fig. 3 (a), the perpendicular component of the external field will induce a supercurrent (yellow arrows), flowing in the YBCO loop to prevent the entrance of the flux. The self-field lines (orange) of the supercurrent are locally stronger at around the constriction and can be detected by using a GMR sensor in the vicinity of the self-field. The YBCO-based mixed sensor has been successfully fabricated using 5 inch sapphire wafer (Fig. 3 (b)). An example of averaged magneto cardiography (MCG) signal using the mixed sensor is shown in Fig. 3 (c), where the QRS complex and T-wave are well visible. The thermal noise level of the device, a few $\mathrm{fT} /(\mathrm{Hz})^{1 / 2}$, is comparable to the noise level of low-Tc SQUID [13].

\section{MR Sensors in CIEDs}

The reed switches have been the most commonly used magnet sensor in CIEDs historically due to its high performance based on mechanical mechanism [14]. However, some drawback of reed switches, including the malfunction caused by compromised hermetically sealed chamber, the tendency of unpredictable behavior both during and after 
exposure to strong magnetic fields such as MRI scanners, their bigger size, and on/off behavior without giving a reading of field strength, have made MR sensor a good candidate to replace reed switches in CIEDs [15].

One example is a GMR multilayer sensor, GF705B, for the application in CIEDs. [16]. As shown in Fig. 4 (a), GF705B is a four terminal sensor that consists for 4 GMR multilayer stripe connected in a Wheatstone bridge configuration. Two of the four sensor stripe are shielded by thick magnetic structure. The typical differential bridge voltage output of the GMR to an external magnetic flux density is shown in Fig. 4 (b). The differential voltage output response is linear between 1.8 and 8 $\mathrm{mT}$, which satisfies the low magnetic sensor requirement of the CIEDs. When the sensor is exposed to higher flux density, the output voltage will maintain in the same level due to the saturation of GMR. Such a characteristic can be used for switching between the differential output mode and the resistance mode. The GMR sensors also has the advantage of occupying a smaller footprint on the substrate of the CIEDs as well as cost reduction compared to the reed switch [15].

\section{MR Sensors for IVD Test}

MR sensors have emerged as one of the promising sensor technology for diagnostic platforms [17]. Depending on the demand in biomedical assays, the MR sensors can be integrated with the diagnostic platforms to detect stray field generated by (a) aggregated magnetic beads in a liquid, (b) magnetic beads immobilized on sensor surface, and (c) magnetic beads on sample surface in a timeof-flight measurement (Fig. 5).

Concerning the approach shown in Fig. 5 (a), a fully automated immunoassay platform, Infinity500, has been developed based on (Teslatronic induced Separation) TIS technology [18]. TIS technology adopts both magnetic and non-magnetic microbeads (much bigger in size), where those beads are suspended in a liquid mixed with a diluted patient sample. Both of the microbeads have a surface functionalization in order to bind specific analyte molecules, such as antigen. If the specific analyte is present in the patient sample, it leads to an aggregation of the magnetic and the non-magnetic beads. In case of a positive sample, a weak stray field will be generated by the magnetic beads because the magnetic beads are embedded in a matrix of non-magnetic beads. In case of a negative sample, a stronger stray field will be generated by the magnetic beads because the magnetic beads are packed in a thin layer with much higher density [19]. A very small difference of magnetic flux density down to $50 \mathrm{nT}$ induced by the two cases mentioned above can be easily detected by using a AMR sensor, AFF787, integrated with the platform [16].

Concerning the approach shown in Fig. 5 (b), there are extensive works reviewed in different resources [2, 5, 17]. Instead of using the microfluidic technique to transport samples and agents, a more simple and reliable way is to integrate a reaction well with the MR sensors [20]. To perform a multiplexed measurement, GMR sensor array was used to detect bioreactions occurred in single microdroplets placed on top of the sensor array. A single sensing element is composed of GMR spinvalve strips connected in parallel and series (Fig. 6 (a)). The strip width and gap between two strips is relevant to the sensitivity of the sensors and therefore has to be optimized. Detection of Femtomolar-level multiplex tumor biomarker has been realized using the GMR sensor-based platform [21]. Such a platform is insensitive to complex matrix in various media and therefore demonstrates that it can be directly to a variety of settings such as molecular biology, clinical diagnostics and biodefense [21].

Concerning the approach shown in Fig. 5 (c), a microfluidic package is necessary to transport samples above the sensor surface for magnetic field sensing. One of the typical application is a magnetic flow cytometry [22] Although the state-of-the-art flow cytometry systems using fluorescence optical detection can provide rapid and reliable for single cells study, the system are bulky, expensive and complex [2]. By integrating magnetophoretic guiding structures with GMR sensor in a single sensor chip, the movement of immunomagnetically labelled single cells through the GMR sensing region can be detected in a time-of-flight measurement (Fig. $7(\mathrm{a})$ ). Figure 7 (b) shows a typical signal output of the GMR sensor induced by single labelled cells. The signal contains the biophysiological information related to the cells such as cell volume, morphological change, and stickiness. Measurement of immuomagnetically labelled single cells in whole undiluted blood has been successfully demonstrated using the the magnetic flow cytometry. The result are benchmarked to state-of-theart optical flow cytometer and Coulter counter instruments [22].

\section{Conclusion}

In this paper we review and outlook MR sensors for some important applications in biotechnology. MR sensors are a promising candidate for detecting biomagnetic signal, performing reliable switching in CIEDs, and realizing versatile biomedical assays for IVD 
test. The low cost, robustness, low power consumption, high sensor performance, and compactness of MR sensors facilitate development of MR sensors-based platform for basic biomedical research as well as products in health care industry.

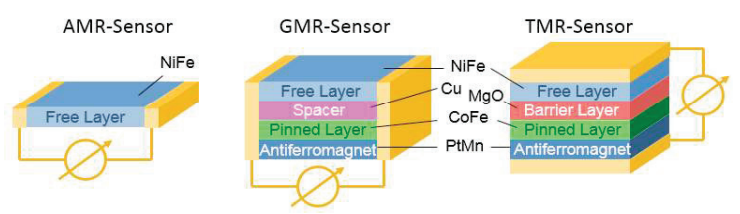

Fig. 1: Construction of $M R$ sensors using AMR, GMR, and TMR effect.

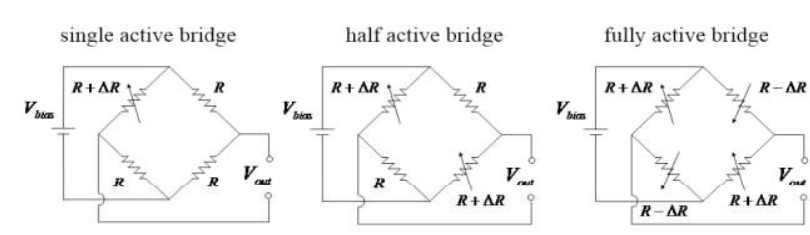

(a)

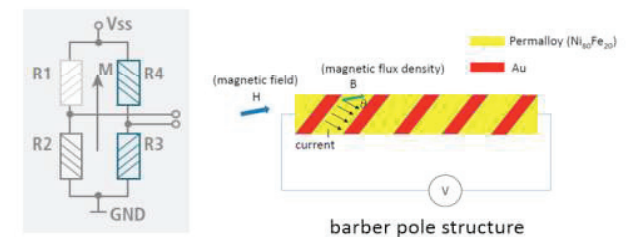

(b)

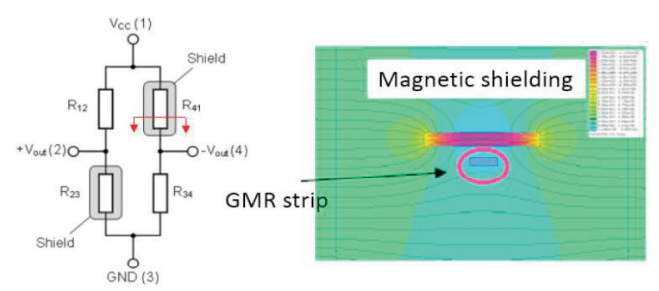

(c)

Fig. 2: $\quad$ MR sensors design: (a) Three types of Wheatstone bridge circuit, (b) barber pole structure design for AMR sensor, and (c) magnetic shielding design for GMR sensor

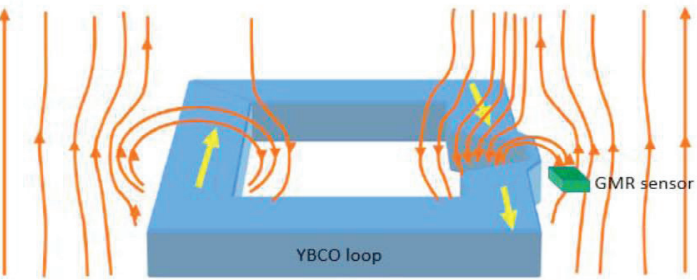

(a)

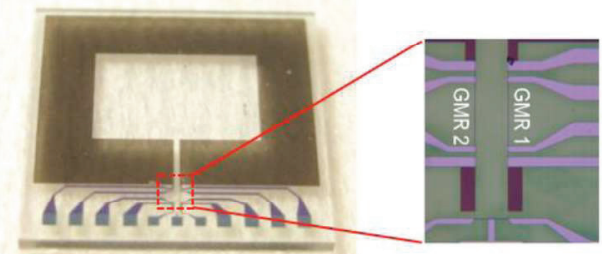

(b)

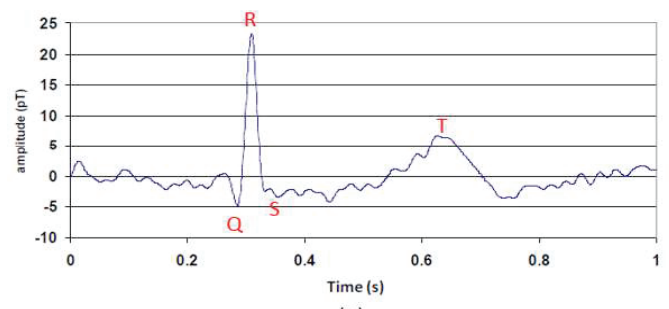

(C)

Fig. 3: (a) The concept of mixed sensor design, (b) fabricated mixed sensor on sapphire substrate, and (c) averaged (30 times) MCG signal, where the QRS complex and T-wave are well visible [13].
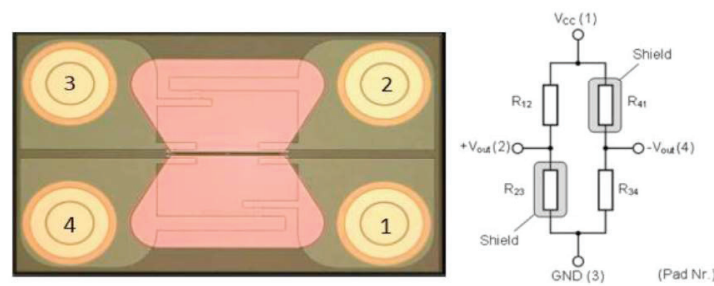

(a)

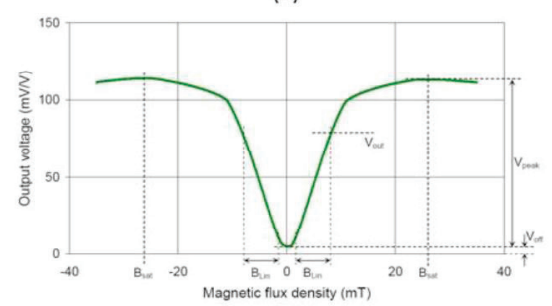

(b)

Fig. 4: (a) GF705B, GMR multilayer sensor and (b) typical output voltage of the GF705 depending on the magnetic flux density [16]. 


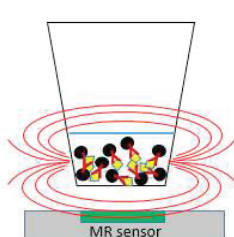

(a)

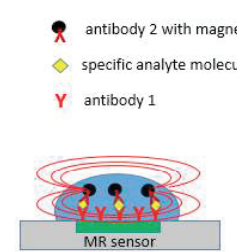

(b)

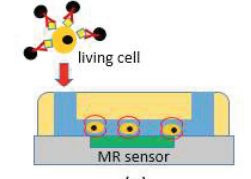

(c)
Fig. 5: Usage of MR sensors-based platform to detect the stray field generated by (a) aggregated magnetic beads in a liquid, (b) magnetic beads immobilized on sensor surface, and (c) magnetic beads on sample surface in a time-of-flight measurement.

A single sensor element

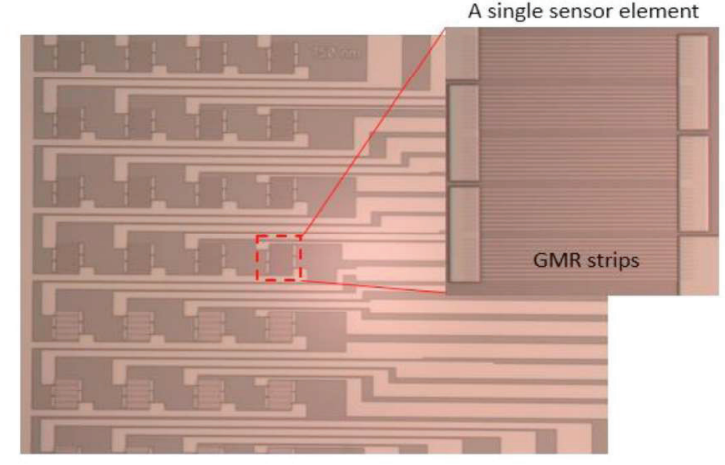

Fig. 6: $\quad$ GMR sensor array for multiplexed measurement. The GMR sensor array is composed of single sensor elements with GMR spin valve strips connected in series and parallel [Courtesy of Todd Klein].

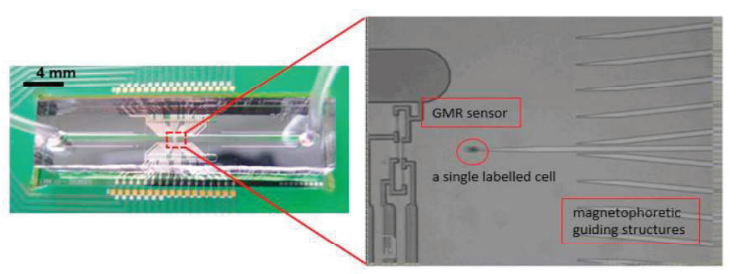

(a)
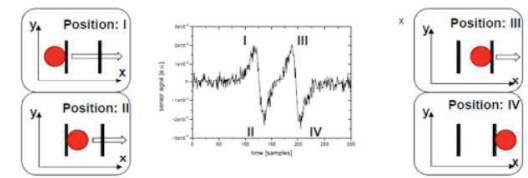

(b)

Fig. 7: A microfluidic sensor chip for the application of magnetic flow cytometry: (a) Time-of-flight measurement of immunomagnetically labelled single cell using a GMR sensor chip with microfluidic package and (b) typical signal output of GMR sensor induced by single labelled cells. [Courtesy of Oliver Hayden].

\section{References}

[1] J.E. Lenz, Proceedings of the IEEE 78, 973-989 (1990); doi: 10.1109/5.56910

[2] P.P. Freitas et al. Lab Chip 12, 546-557 (2012); doi: 10.1039/c1lc20791a

[3] S. Jacob et al. Europace 13, 1222-1230 (2011)

[4] D. A. Healy, C. J. Hayes, P. Leonard, L. McKenna, R. O'Kennedy, TRENDS in Biotechnology 25, 125-131(2007): doi: http://dx.doi.org/10.1016/j.tibtech.2007.01.0 04

[5] A. van Reenen, A. M. de Jon, J. M. J. den Toonder, M. W. J. Prins, Lab Chip 14, 19661986 (2014); doi: 10.1039/c3lc51454d

[6] W. Thomson, Proc. R. Soc. Lond. 8, 546-50 (1856)

[7] M. Julliere, Phys. Lett. 54A, 225-226 (1975); doi: 10.1016/0375-9601(75)90174-7.

[8] G. Binasch, P. Grünberg, F. Saurenbach, W. Zinn, Phys. Rev. B 39, 4828 (1989); doi: 10.1103/PhysRevB.39.4828

[9] M. N. Baibich et al., Phys. Rev. Lett. 61, 2472 (1988); doi: 10.1103/PhysRevLett.61.2472

[10] S.S.P. Parkin, N. More, K.P. Roche, Phys. Rev. Lett 64, 2304-2307 (1990); doi: 10.1103/PhysRevLett.64.2304

[11] S.S.P. Parkin; et al. Nat. Mat. 3, 862-867 (2004); doi: 10.1038/nmat1256

[12] M. Pannetier-Lecoeur, C. Fermon, G. Le Goff, J. Simola, E. Kerr, Science 304, 1648-1650 (2004);doi: 10.1126/science.1096841

[13] M. Pannetier-Lecoeur, Condensed Matter, Universite Pierre et Marie Curie - Paris VI, (2010)

[14] Reed Switch Characteristic.: http://www.misensor.info/reed-switch/reedswitch-characteristics

[15] E. N. Irudhayaraj, H. krätschmer, J. von Arx, L. Stotts, 13th Symposium Magnetoresistive Sensors and Magnetic Systems, 23-30 (2015)

[16] Sensitec $\mathrm{GmbH}$, www.sensitec.com

[17] D. L. Graham, H. A. Ferreira, P. P. Freitas, TRENDS in Biotechnology 22, 455-462(2004); doi:10.1016/j.tibtech.2004.06.006

[18] ORGENTEC Diagnostika GmbH http://orgentec.com/de/50-systems/infinity

[19] V. Kirste, 13th Symposium Magnetoresistive Sensors and Magnetic Systems, 31-35 (2015)

[20] W. Wang, Yi Wang, L. Tu, Y. Feng, T. Klein, J.P. Wang, Scientific Reports 4, 5716 (2014); doi:10.1038/srep05716

[21] R. S. Gaster et al., Nature Medicine 15, $1327-$ 1332 (2009); doi: 10.1038/nm.2032

[22] M. Helou et al., Lab Chip 13, 1035-1038 (2013); doi: 10.1039/C3LC41310A 